\title{
Analyse de l'appropriation d'un parcours pédagogique numérique par un formateur : le cas du dispositif Pairform@nce
}

Analysis of how a teacher adapts an online educational scenario: the case of the Pairform@nce programme

Eugénie Duthoit et Stéphanie Mailles-Viard Metz

\section{OpenEdition}

Journals

Édition électronique

URL : http://journals.openedition.org/activites/203

DOI : 10.4000/activites.203

ISSN : $1765-2723$

Éditeur

ARPACT - Association Recherches et Pratiques sur les ACTivités

\section{Référence électronique}

Eugénie Duthoit et Stéphanie Mailles-Viard Metz, « Analyse de l'appropriation d'un parcours

pédagogique numérique par un formateur : le cas du dispositif Pairform@nce »,Activités [En ligne],

9-1 | Avril 2012, mis en ligne le 30 mars 2012, consulté le 19 avril 2019. URL : http://

journals.openedition.org/activites/203 ; DOI : 10.4000/activites.203

\section{(c) $\oplus \Theta \Theta$}

Activités est mis à disposition selon les termes de la licence Creative Commons Attribution - Pas d'Utilisation Commerciale - Pas de Modification 4.0 International. 


\title{
Analyse de l'appropriation d'un parcours pédagogique numérique par un formateur : le cas du dispositif Pairform@nce
}

\author{
Eugénie Duthoit \\ Laboratoire PRAXILING CNRS UMR 5267, Université Paul-Valéry Montpellier 3 \\ Route de Mende, 34199 Montpellier Cedex 5 - eugenie.duthoit@ univ-montp3.fr

\section{Stéphanie Mailles-Viard Metz} \\ Laboratoire PRAXILING CNRS UMR 5267, Université Paul-Valéry Montpellier 3 \\ Route de Mende, 34199 Montpellier Cedex 5 - stephanie.metz@univ-montp3.fr
}

\begin{abstract}
Analysis of how a teacher adapts an online educational scenario: the case of the Pairform@nce programme. This paper sets out to analyse the use of online resources and training programmes by a teacher who was not the designer. From theoretical considerations about the design process and some views on the use of resources, we attempt to highlight the relevant appropriation processes using indicators that show instrumentation and instrumentalisation procedures (based on pragmatic, epistemic, heuristic and cooperative mediation). Our research focused on a French online further education programme for teachers called Pairform@nce). More specifically, we look at the alteration, addition and deletion of resources, as well as scenario changes and the use of back-up material as indicators of appropriation. These indicators are proposed following the analysis of four interviews with teachers, and were verified by reading other teachers' logbooks. As a result of the analysis, we suggest a cooperative system for improving asynchronous interaction between designer and teacher.
\end{abstract}

\section{KEYWORDS}

appropriation, online further education, training, designer, teacher, cooperation.

\section{1.- Introduction}

L'intégration des Technologies de l'Information et de la Communication dans l'Enseignement (TICE), prônée, notamment par le décret du 11 juillet 2006 et le BO n 29 , implique une nouvelle organisation du travail des enseignants. Cette transformation des pratiques peut être prise en charge entre autres dans le cadre de la formation continue, par des formateurs. La complexité d'approche de l'outil technologique modifie le schéma d'organisation traditionnel d'une formation qui se construit souvent sur une interaction formateur-enseignant (également appelé stagiaire).

Ici, la mutualisation des ressources et des scénarios pédagogiques permise par les technologies de l'information et de la communication met en jeu l'implication d'un nouvel acteur : le concepteur. Ce dernier propose un scénario pédagogique qui intègre la mise en 
scène d'outils. Le scénario est ensuite repris par le formateur. Nous entendons par scénario pédagogique, un ensemble de ressources organisé de façon à proposer aux enseignants une formation collaborative intégrant l'usage des TICE et de manière générale, un scénario pédagogique est «le résultat du processus de conception d'une activité d'apprentissage » (Brassard \& Daele, 2003, p.2). Par ailleurs, la conception d'un scénario pédagogique s'inscrit dans un dispositif d'apprentissage que nous nommerons, en ce qui concerne la formation continue, un dispositif de formation. La notion de dispositif hybride intègre non seulement le matériel et la technologie, mais également le symbolique et le relationnel (Charlier, Deschryver \& Peraya, 2006).

Notre étude se situe dans l'interaction asynchrone et indirecte entre le formateur et le concepteur. En effet, le concepteur peut s'adresser au formateur par le biais du scénario qu'il propose et peu de systèmes proposent un retour de sa mise en œuvre. Dans l'autre sens, le formateur s'interroge sur le scénario proposé et ne peut pas toujours s'adresser directement au concepteur. Ainsi, des outils doivent être conçus pour mettre en interaction directe ou indirecte ces deux acteurs dont les tâches diffèrent et se complètent. Ces difficultés de communication ne sont pas sans conséquence sur la qualité des formations : les ressources prescrites par le concepteur risquent d'être peu utilisées, car parfois peu intégrables (incompréhension, contexte varié...) par le formateur. Il peut donc exister un écart entre ce qui est prescrit et ce qui est réalisé (Mailles-Viard Metz, 2009 ; Michel, Garrot, \& George, 2007).

Nous nous intéressons ainsi aux activités de ces acteurs de la formation continue afin de proposer une méthode de traçabilité qui permettrait de rendre compte de leurs activités. Cette méthode pourra laisser envisager un moindre écart entre l'usage prescrit et l'usage effectif des ressources associées aux parcours de formation en amont par le formateur et, en aval, une amélioration des nouveaux schémas d'organisation proposés aux enseignants. L'objectif est ainsi de proposer un point de vue didactique et ergonomique à propos de la conception et l'usage de scénarios pédagogiques mutualisés.

Tout d'abord, nous exposons notre cadre théorique qui porte sur l'activité d'appropriation d'un dispositif instrumenté. Notre problématique pose les hypothèses que nous testons dans une situation de formation continue en ligne des enseignants du second degré. L'analyse de quatre entretiens, puis celle de journaux de bord de formateurs nous permet de proposer des indicateurs de l'appropriation spécifiques à cette situation. Nous concluons par la proposition de développement d'un outil permettant d'établir une communication indirecte entre le concepteur et le formateur, l'interaction étant elle-même mobilisatrice de traces et donc représentative de l'appropriation puisqu'elle "s'inscrit dans une activité, un environnement social et professionnel, ainsi que dans un processus d'acquisition de connaissance ». (Cotreaux \& Jacques, 2004, p. 2)

\section{2.- Interroger le processus de l'appropriation d'un dispositif instrumenté}

\section{1.- Appropriation et apprentissage}

Dans le langage courant, le terme «appropriation» se définit par «ce qui appartient en propre » au sujet de l'activité. Le processus peut s'appliquer à la fois à un artefact matériel (outil, technologie, etc.) ou à un artefact symbolique (idée, scénario, etc.). En tant qu'élément central du processus d'appropriation, nous considérons l'artefact (ou outil) comme le résultat d'une activité finalisée (Rabardel, 1995) du concepteur qui s'est imaginé un usage futur de celui-ci. L'artefact peut être, toujours selon Rabardel, soit objet, en tant qu'objet de l'activité (par exemple dans les activités de dépannage, maintenance), soit moyen, en tant que ressource mobilisée ou mobilisable de l'activité. 
L'utilisation en constante augmentation ces dernières années des TICE suscite une littérature de plus en plus abondante. Le processus d'appropriation est souvent défini compte tenu des conditions à son déroulement effectif : soit «maitrise cognitive et technique », «intégration sociale» et «potentialité de création» (Proulx, 2001, p. 2), ou en fonction «des caractéristiques et des objectifs de chaque situation de travail » (Cuvelier \& Caroly, 2009, p. 15). De manière générale, l'ensemble des facteurs inhérents au processus d'appropriation sont régulièrement mis en valeur : «L'acte d'appropriation se révèle, non pas par rapport à l'interface en elle-même, mais en dehors, dans une combinatoire d'éléments disparates et complémentaires. Cet ensemble d'actants de l'environnement cognitif de l'usager se définit dans un mouvement qu'il associe à la recherche d'une meilleure optimisation et rationalisation de sa pratique » (Cotreaux \& Jacques, 2004, p. 9) ainsi que le caractère dynamique et continu du processus : «L'appropriation d'une stratégie opératoire est un processus continu de développement » (Cuvelier \& Caroly, 2009, p. 15).

Enfin, Theureau (2011) précise ces définitions précédentes du processus en y voyant une « intégration, partielle ou totale, d'un objet, d'un outil ou d'un dispositif à la culture propre de l'acteur, accompagnée (toujours) d'une individuation de son usage et (éventuellement) de transformations plus ou moins importantes de cet objet, de cet outil ou de ce dispositif luimême » (p. 11).

L'appropriation est, en effet, vue en tant que dynamique à la fois sociale et individuante : «l'un des enjeux de l'appropriation serait une co-construction interactionnelle d'une situation, considérée comme l'actualisation de modalités précises, explicites et négociées de l'implication des composantes artefactuelles et objectales dans l'organisation de l'action » (Paquelin, 2009, p. 191). Ici, l'appropriation est vue comme double: intégrant à la fois l'apprentissage des fonctionnalités du dispositif et l'apprentissage des contenus: «on y passe de l'idée d'appropriation à celle d'apprentissage et de confrontation entre des formes de connaissances différentes, mais qui sont articulées au sein d'un même processus » (Béguin, 2007, p. 7).

Les principales théories de l'apprentissage reconnaissent l'importance de l'expérience de l'individu dans le processus d'apprentissage. De même, les analyses existantes du processus d'appropriation visent à s'attacher à l'activité d'un sujet face à un artefact. Dans les deux cas, l'artefact peut être matériel ou symbolique. Quelle est donc la différence entre « appropriation » et « apprentissage »?

Définir l'appropriation d'un scénario pédagogique nous semble alors particulièrement nécessaire afin de caractériser les activités d'un formateur qui utilise et donc s'approprie un scénario conçu par un autre. Dans le contexte particulier de la formation continue et dans le champ de la didactique professionnelle (Pastré, 2005), l'appropriation peut être différenciée de l'apprentissage par les dimensions de l'activité auxquelles elle fait appel; si l'apprentissage est surtout perçu comme une activité productive par le formateur en tant que finalité pour les stagiaires dont il a la charge, finalité liée à son activité de formateur, l'appropriation du scénario pédagogique intègre davantage la dimension constructive de l'activité du formateur puisqu'il s'approprie un scénario pédagogique mutualisé pour mettre en œuvre sa formation. "L'activité constructive concerne, d'une part, les instruments dans leurs composantes psychologiques et matérielles, d'autre part, les compétences et notamment les conceptualisations développées par et pour l'activité »(Samurçay, 2005, p. 66).

Notre analyse s'inscrit dans une double dimension :

- Sur un niveau macro, dans le cadre d'une démarche d'analyse de l'activité pour la conception : la prise en compte de l'appropriation dans la conception et la préconisation de retours d'usage, implique d'appréhender celle-ci non seulement selon les critères d'utilisabilité (une mise en œuvre face aux propriétés de l'artefact), mais également d'utilité (en tant que besoin de l'activité dans un contexte donné) : "L'évolution de nos 
problématiques nous amène à envisager une évolution de notre pratique par une évolution qualitative de l'utilité et l'utilisabilité (par exemple en intégrant la dimension contexte), mais aussi par l'explicitation de critères qui n'organisent pas encore notre pratique » ( Haradji \& Faveaux, 2006, p. 26).

- Sur un niveau micro, dans le cadre d'une mise en valeur d'indicateurs du processus d'appropriation afin d'analyser l'activité spécifique d'un formateur: une analyse de la genèse instrumentale (Rabardel, 1995) nous a semblé intéressante. En effet, cette prise en compte de la genèse instrumentale (ou des genèses instrumentales en vigueur) pendant l'appropriation amène à s'intéresser de manière plus particulière à la fois au formateur et au scénario pédagogique. Par ailleurs, l'inscription des genèses instrumentales dans le travail de conception est destinée à inscrire des possibilités multiples au sein des artefacts.

L'usage prend ainsi forme dans des trajectoires d'usages, réinvesties selon les contextes. Dans cette dynamique, la mesure d'un «écart» entre le prescrit et l'effectif ${ }^{1}$ fait également émerger une volonté d'analyser ces nouveaux usages dans le contexte particulier des dispositifs de formation.

L'écart entre prescrit et réel, dans les situations d'apprentissage ou de formation (qu'il s'agisse d'une tâche ou de l'usage d'un scénario pédagogique), pose question : "Une tâche prescrite ou objective est relativement extérieure à l'opérateur : c'est ce qu'on lui "demande de faire" ou ce qu" "il y a à faire". On oppose généralement cette tâche à la tâche effective, c'est-à-dire à ce que fait réellement l'opérateur humain. Car, l'utilisateur interprète la tâche: cette représentation cognitive de la tâche par l'opérateur humain peut être (est) sensiblement différente de la représentation que l'on pourrait s'en faire en analysant rationnellement la situation» (Tricot \& Nanard, 1998 p. 3). En effet, si cet écart est constructif, l'objectif d'apprentissage ou de formation nécessite qu'il ne soit pas trop important.

\section{2.- Appropriation et genèse instrumentale}

Afin d'observer l'appropriation dans ce contexte très précis, nous nous inscrivons tout particulièrement dans le cadre théorique de la genèse instrumentale de Rabardel (1995). Ce dernier offre des outils conceptuels intéressants pour une étude de l'appropriation d'un artefact, technique et/ou symbolique.

Pour Rabardel (1999, p. 15) l'instrument en tant qu'objet matériel, production concrète « contient, sous une forme spécifique l'ensemble des rapports que le sujet peut entretenir avec la réalité et dans laquelle il permet d'agir». Ces rapports se définissent selon deux composantes de l'instrument : l'artefact, c'est-à-dire, l'instrument tel qu'il apparait aux yeux du sujet et les schèmes d'utilisation, c'est-à-dire tout ce qui résulte de la construction propre au sujet : les schèmes sociaux préexistants et les schèmes d'usage (Figure 1). L'instrument se construit ainsi dans l'usage et les contextes et n'est jamais figé. Rabardel (1995) distingue ainsi deux rapports principaux entre les hommes-sujets et les artefacts technologiques (ou symboliques) :

— L'artefact est objet de l'activité, notamment dans les activités de dépannage, maintenance, contrôle ;

— L'artefact est moyen, ressource mobilisée ou mobilisable de l'activité, c'est ainsi le cas des scénarios pédagogiques mutualisés ;

- Cette approche instrumentale nous intéresse ainsi à double titre. D'une part, elle permet de définir l'activité du formateur. En effet, dans le cadre de cette activité, l'instrument devient le support de médiations instrumentales définies par Rabardel (1995) :

- La médiation épistémique : l'instrument est un moyen qui permet la connaissance de

$1 \mathrm{Ou}$ «état prescrit », «état perçu », « état prévu », « état vécu » (Paquelin, 2009) 
l'objet de l'activité ;

- La médiation pragmatique : l'instrument est moyen d'une action transformatrice dirigée vers l'objet de l'activité ;

- La médiation heuristique : l'instrument est moyen d'une action transformatrice dirigée vers le sujet même ;

- La médiation collaborative : l'instrument est moyen d'une action transformatrice dirigée vers l'action de l'autre sujet.

D'autre part, le formateur s'approprie l'artefact en lui conférant des fonctions qui vont audelà de ses fonctions constituantes, de ses potentialités à travers des activités de genèse instrumentale, envisagées par le biais de deux processus : l'instrumentation ou l'adaptation du sujet aux contraintes que constituent l'artefact et ses fonctions constituantes et l'instrumentalisation ou la transformation (enrichissement ou spécification) de l'artefact ou d'une partie de l'artefact afin d'en faire un moyen pour l'activité. La combinaison de ces deux processus conduit à la réorganisation d'une partie des schèmes d'utilisation et à la modification de l'instrument.

L'appropriation au même titre que la genèse instrumentale, est ainsi un processus en train de se faire donc dans un continuum inscrit à la fois dans une temporalité longue (temps de la vie) et dans une temporalité brève (temps de l'activité) du sujet. Le sujet s'approprie l'artefact en s'appuyant sur le monde extérieur et les préconstruits sociaux (les méthodes, les concepts, les normes, les genres des collectivités auxquelles il appartient), il développe son pouvoir d'agir. Les ressources sont ainsi déterminantes pour «être capables d'agir» et «pouvoir agir » dans ces structures sociales. Rabardel (2005) propose de conceptualiser les rapports entre capacité et pouvoir, c'est-à-dire la différence entre ce qui est mobilisable, dans une situation donnée, par le sujet et ce qui est effectivement possible, ce qui est au pouvoir du sujet, dans la singularité de la situation. La capacité d'agir est liée à "l'ensemble des ressources développées comme moyens potentiellement opératifs dans le monde où ils peuvent être mobilisés et mis en cuvre par les sujets». Le pouvoir d'agir «se situe par rapport à un rapport singulier au monde réel, rapport qui actualise et réalise la capacité d'agir en en transformant les potentialités en pouvoir» (Rabardel, 2005, p. 19). L'interaction entre le concepteur et le formateur se construit alors à partir (1) de l'utilisation ou non des ressources mobilisables et (2) des ressources mobilisées et donc du décalage entre la capacité d'agir mobilisée par le concepteur sur le scénario proposé dans son rapport indirect au formateur et du pouvoir d'agir effectif du formateur dans son propre contexte.

L'appropriation d'un artefact prend donc deux dimensions qui se complètent et s'interfèrent :

- Une appropriation individuelle qui se définit, dans le cadre de l'approche instrumentale de Rabardel par la conclusion de l'instrumentation et de l'instrumentalisation de l'artefact ;

- Une appropriation collective qui se coordonne à l'appropriation individuelle, mais s'inscrit dans le monde et le contexte temporel qui entourent le sujet.

L'appropriation est, ainsi, un processus dialogique entre le sujet et l'objet d'appropriation (dans le cadre de cette étude, les parcours de formation) qui :

- en fonction du contexte, est inscrit dans une temporalité plus ou moins longue par une évolution et un enrichissement non seulement de l'artefact qui devient instrument au sens de Rabardel (1995) ;

— en fonction du sujet qui évolue lui-même selon cette genèse instrumentale et des schèmes qu'il rencontre, traduit et s'approprie pendant le processus.

Si la théorie de la genèse instrumentale nous permet de décrire le processus d'appropriation, l'appropriation nous apparait alors comme une (ou plusieurs) genèse(s) instrumentale(s), en cours d'accomplissement, inscrite(s) dans une temporalité spécifique: "De plus, le 
processus d'appropriation, d'une part, du fait de sa durée, passe par des épisodes disjoints d'une même pratique à travers le temps» (Theureau, 2011, p. 5).

\section{3.- Vers une définition du processus d'appropriation : laisser libre cours à l'adaptation sans dénaturer l'artefact prescrit}

Nous voyons ainsi l'appropriation d'un parcours de formation comme une genèse instrumentale (Rabardel, 1995) «en cours d'accomplissement» d'un parcours de formation intégrée dans une temporalité variable et également comme un processus situé (Cuvelier \& Caroly, 2009). L'appropriation peut ainsi être évaluée en fonction d'un écart entre ce qui est prescrit et ce qui est réalisé dont la valeur dépendrait de la temporalité dans l'usage, de l'expertise du formateur et d'un contexte d'appropriation spécifique. Ces différents éléments nous montrent que les ressources proposées doivent être propices à une instrumentalisation du scénario de formation prescrit. Le scénario doit aussi laisser libre cours au formateur de s'adapter aux contraintes que constituent les différents contextes d'appropriation dans lequel il est susceptible d'enseigner. En effet, le potentiel d'appropriation donne une liberté de reconception du scénario au formateur.

Nous nous posons ici deux questions : la première concerne la mesure de l'appropriation et la seconde les moyens nécessaires pour la faciliter.

Pour répondre à notre première question et au regard des éléments énoncés précédemment, l'appropriation semble donc pouvoir se définir en fonction de l'écart entre l'usage prescrit et l'usage effectif du parcours de formation associé à la temporalité d'usage (ou de formation dans le cas qui nous intéresse). De plus, si l'écart entre l'usage prescrit et l'usage effectif est signifiant dans l'analyse de l'appropriation, un seuil semble nécessaire afin de ne pas dénaturer l'artefact et donc de ne pas détourner les objectifs d'apprentissage. Toutefois, la prise en compte de cet écart et des catachrèses du formateur dans la conception des scénarios pédagogiques pourrait aider l'appropriation de ces dernières et aider à "considérer les catachrèses en tant qu'indices du fait que les utilisateurs contribuent à la conception des usages des artefacts » (Rabardel, 1995, p. 124).

La réponse à la deuxième question est pour nous liée entre autres à une interaction indirecte entre le concepteur et le formateur. Le concepteur doit rendre explicite la formation pour qu'elle soit comprise par le formateur, première étape dans l'appropriation du formateur. Il peut s'agir d'assistants au sens de Trouche (2008). Cependant, le concepteur n'aura de retour de l'efficacité de ses ressources que si le formateur l'informe de sa façon de mettre en œuvre la formation. Notre hypothèse est donc que la réalisation d'un Journal de Bord par le formateur permettra cette interaction et sera un retour d'usage pour le concepteur.

\section{4.- Une étude de cas : un dispositif hybride de formation professionnelle des enseignants du second degré}

\section{1.- Pairform@nce : un programme de formation continue hybride}

Pour vérifier ces hypothèses théoriques, nous avons analysé des expériences d'appropriation de parcours de formation proposés à des formateurs par le récent programme de formation Pairform@nce dont l'objectif premier est l'enrichissement des pratiques pédagogiques des professeurs du premier et second degré, notamment dans le cadre des mesures de validation du C2i enseignant ${ }^{2}$. Ce programme permet à des concepteurs de créer des parcours de formation. Un parcours de formation désigne le scénario proposé, c'est-à-dire l'ensemble des ressources et des étapes proposées aux formateurs extérieurs pour mettre en œuvre une

2 Certificat Informatique et Internet de niveau 2 pour les enseignants a été crée en 2004. 
formation intégrant l'usage de nouvelles technologies sur un sujet ou un domaine particulier (Soury-Lavergne \& Sanchez, 2009). Ces parcours sont récupérés puis adaptés par des formateurs qui assurent des formations auprès de stagiaires dans les différentes académies. Dans ces formations, l'accent est mis sur l'appropriation des TICE dans des contextes disciplinaires différents, non seulement dans le contenu, mais aussi dans l'approche pédagogique : les formations proposées sont hybrides, mêlant séances de travail en présence et à distance. Cette situation doit nous permettre de vérifier nos hypothèses dans le sens où les parcours pédagogiques sont mutualisés, dans le cadre d'une communauté de pratique afin de favoriser une démarche d'apprentissage collaboratif. En effet, ce programme repose sur l'implication de trois acteurs :

- Le concepteur : en tant qu'individu ou en équipe, il est engagé dans la production du parcours de formation et conçoit l'aspect technique de sa mise en œuvre. Le cahier des charges stipule qu'il est destinataire des retours d'usage des parcours pendant les deux années scolaires qui suivent sa mise en œuvre. Il arrive souvent que le concepteur soit luimême formateur et mette à disposition des parcours qu'il a conçus.

- Le formateur : il met en ouvre le parcours de formation en constituant les équipes de professeurs stagiaires en formation continue et anime, donne vie à l'utilisation de ce parcours par l'équipe de stagiaires. Il arrive qu'il soit aussi le concepteur des parcours qu'il met en œuvre.

— L'enseignant appelé stagiaire : il produit en équipe une situation pédagogique à partir du suivi d'un ou plusieurs parcours et tente d'intégrer des éléments de la formation dans ses pratiques d'enseignement et dans son cadre scolaire habituel.

Au-delà du programme, la mise en œuvre d'une plateforme nationale intègre autant les acteurs que les technologies, nous l'appréhendons alors en tant que dispositif. Ce dispositif prend également une valeur institutionnelle puisque les parcours de formation sont proposés dans le cadre d'un Plan Académique de Formation (PAF). L'organisation du dispositif en ligne est nationale, mais l'organisation des formations se fait au niveau académique.

La conception et l'appropriation d'un parcours de formation s'inscrivent dans ce dispositif très spécifique qu'est Pairform@nce. L'artefact correspond au scénario proposé par un concepteur ; il est approprié dans le cadre du dispositif de Formation Ouverte et à Distance (FOAD) (Pairform@nce), mais également selon le contexte d'appropriation du formateur. La prescription pourrait s'appréhender dès la conception, mais ce n'est pas ici la question.

Le scénario prescrit se situe dans le dispositif Pairform@nce (ne serait-ce que par le cahier des charges) et respecte un certain nombre de règles. Par ailleurs, il se caractérise par des ressources à utiliser (pour le formateur seul ou ses enseignants) et des outils.

Dans cette étude, ce sont principalement les activités du concepteur et du formateur face à un même parcours qui sont observées dans la mesure où celles-ci permettent de mettre en lumière l'écart entre usage prescrit et usage effectif et donc l'instrumentalisation et l'instrumentation des parcours.

\section{2.- Méthode globale}

Rappelons que nos hypothèses concernent la définition puis les modalités de facilitation de l'appropriation. Le premier objectif tente donc d'identifier des indicateurs de l'appropriation à partir de notre proposition de définition : «L'appropriation peut être évaluée en fonction d'un écart entre ce qui est prescrit et ce qui est réalisé dont la valeur dépendrait de la temporalité dans l'usage sans pour autant dénaturer le prescrit». Comment trouver des indicateurs de l'appropriation dans l'explicitation de leurs activités par les formateurs dans la situation Pairform@nce?

Pour mener à bien cette analyse, nous avons procédé en deux étapes :

- Analyse préliminaire : une analyse de quatre entretiens semi-directifs afin, d'une part, de 
mieux connaître la situation étudiée, et d'autre part, de faire émerger des indicateurs de l'écart entre usage prescrit et l'usage effectif des parcours.

- Analyse principale: une analyse de journaux de bord papiers remplis par d'autres formateurs est réalisée afin de valider nos indicateurs d'une part et observer de manière plus fine l'appropriation des parcours de formation d'autre part pour montrer si l'interaction formateur - concepteur peut faciliter l'appropriation par le formateur.

\section{5.- Analyse préliminaire : entretiens semi-directifs}

\section{1.- Méthode spécifique}

L'observation concerne donc quatre entretiens individuels semi-directifs avec des formateurs à partir d'un guide d'entretien. Après avoir demandé au formateur de se présenter, de décrire son parcours de formateur et son expérience dans le cadre de Pairform@nce, on se focalise sur sa mise en œuvre d'un parcours de formation. Pendant l'entretien, nous posons la question des facteurs contextuels et les choix personnels qui ont pu influencer la modification, l'ajout ou la suppression de données du parcours et/ou l'utilisation d'outils proposés dans ce dernier. Les questions sont adaptées au fur et à mesure de l'entretien.

Trois des quatre entretiens ont été menés en visioconférence à l'aide de l'outil FlashMeeting ${ }^{3}$ qui permet non seulement la transcription des entretiens, mais également une régulation de la parole grâce à l'option « prendre/rendre la parole ${ }^{4}$ ». Cette option nous a semblé intéressante dans la mesure où le formateur est libre de s'exprimer plus ou moins longuement sans attendre une réaction de la part de celui qui mène l'entretien. De fait, on trouve ici un outil permettant de présenter la trame du guide d'entretien au formateur et ainsi de cadrer son discours dans une attitude non directive. En effet, c'est l'interviewé qui rend la parole, en cliquant sur l'option sous l'écran. Le quatrième entretien a été effectué par téléphone et n'a pas été enregistré, seules des notes ont été prises.

Les entretiens ont duré entre vingt et trente minutes chacun et leur déroulement a varié selon les réponses des formateurs dès lors que leurs expériences et leur appropriation des parcours dans le cadre Pairform@nce se sont révélées différentes. Cela a marqué grandement notre intérêt dans le cadre de l'étude des processus d'appropriation de parcours de formateur selon les préconisations du programme Pairform@nce. En effet, les contextes dans lesquels les parcours ont été appropriés ainsi que les profils des formateurs interviewés diffèrent :

- Le premier formateur (F1) est également concepteur de parcours et fortement impliqué dans la mise en œuvre du programme Pairform@nce. Il a déjà conçu puis mis en œuvre un parcours de formation.

— Le deuxième formateur (F2) interviewé est également impliqué dans le programme Pairform@nce par le biais d'une action de recherche et a participé à une formation de formateur en amont. Suite à cette formation, il a mis en œuvre un parcours de formation dont il n'est pas le concepteur.

— Le troisième formateur $(\mathrm{F} 3)$ a mis en œuvre un parcours de formation dont il n'est pas le concepteur suite à une demande d'un Inspecteur de l'Éducation Nationale (IEN). À l'inverse des formateurs F1 et F2, il n'a ni conçu de parcours ni participé à une action de recherche en amont de la mise en œuvre du parcours, mais s'est engagé dans la conception d'un parcours à la suite de cette mise en œuvre.

— Le quatrième formateur (F4) interviewé par téléphone a pris la suite du concepteur du parcours qui n'avait pas la possibilité de faire la formation. Sans connaissance du

\footnotetext{
3 http://fm-openlearn.open.ac.uk/

4 《Start broadcasting »
} 
programme Pairform@nce ni des formations intégrant les TICE, il met en œuvre ce parcours en s'appuyant exclusivement sur les exemples proposés pour se faciliter la tâche.

Les formateurs (F2-F3-F4) ont été choisis parce qu'ils ont mis en œuvre dans leur académie une formation qu'ils n'ont pas eux-mêmes conçue. Cet aspect s'est avéré une difficulté dans la recherche de formateurs, dans la mesure où les parcours sont souvent mis en œuvre par des formateurs qui sont, eux-mêmes, concepteurs au sein du programme Pairform@nce. Le formateur $\mathrm{F} 1 \mathrm{a}$, dès les débuts du projet, conçu une formation destinée à être appropriée par d'autres formateurs.

En revanche, dans ce cadre très particulier de Pairform@nce où la conception d'un parcours est régulée par un cahier des charges précis; l'implication dans la mise en œuvre du programme ou la conception d'un parcours implique une connaissance du dispositif que nous qualifions d'expertise.

L'enjeu de la grille d'entretien a donc été de savoir comment a été menée l'appropriation du parcours et si des modifications ont été apportées au parcours pour sa mise en œuvre dans le cadre de la formation. Les entretiens ont ensuite fait l'objet d'une analyse fondée sur les discours des formateurs et plus particulièrement à partir du traitement des marqueurs linguistiques (pronoms personnels, modalisateurs, adverbes, construction syntaxique) puis d'un traitement thématique qui nous a permis de faire émerger des indicateurs de l'appropriation des parcours.

\section{2.- Résultats de l'analyse des entretiens}

Les quatre entretiens mettent l'accent sur les données et contraintes organisationnelles et humaines qui auraient une influence non négligeable sur la mise en œuvre du parcours. Les formateurs, dans les différents entretiens, ont mis en valeur des variables externes à leur activité en tant que facteurs importants au déroulement de l'appropriation des parcours de formation. De fait, ils associent les modifications apportées au parcours de formation à une adaptation rendue nécessaire par des contraintes organisationnelles clairement définies. Ces contraintes s'expriment par l'utilisation de verbes d'obligation, "nous avons été obligés de réduire le présentiel pour des histoires de coup de déplacement» (F3). De la même façon, l'utilisation de tournures au gérondif et de groupes prépositionnels permettent aux formateurs d'exprimer clairement l'idée que les contraintes organisationnelles ont un impact sur la modification des parcours : "Alors en fait euh en ce qui concerne déjà le calendrier euh, on s'est inspiré du calendrier en l'adaptant aux dates de nos formations et on a précisé les dates, on l'a [comment dire] un petit peu complété à ce niveau là pour que les stagiaires aient un repère précis par rapport aux dates qui sont des dates quand même butoirs » (F2) et "Nous avons aménagé le calendrier en fonction du temps disponible des stagiaires, car c'est un stage long» (F3). Ici, l'aspect temporel est exprimé comme déterminant dans l'appropriation du parcours de formation. À partir de ces remarques, nous avons identifié quatre facteurs externes du processus d'appropriation d'un parcours de formation par un formateur :

— Les facteurs techniques ;

— Le facteur temporel ;

— Les facteurs humains : gestion des groupes et des stagiaires impliqués dans la formation et juridiques (droit d'auteurs des ressources, etc.) ;

— Les facteurs liés au contexte : gestion des moyens disponibles compte tenu d'un budget alloué.

Néanmoins, la démarche d'appropriation des quatre formateurs interrogés se différencie par leur évaluation personnelle du parcours associée à leurs parcours professionnels différents.

«Les ressources que j'ai utilisées euh et qui ne sont pas dans le parcours bah sont des ressources personnelles parce que j'ai eu à travailler avec des enseignants spécialisés et 
donc je travaille aussi de façon assez importante la différenciation et donc automatiquement ce que j'utilise en différenciation je l'ai réinjecté notamment pour commenter le diaporama qui est présent dans le parcours le diaporama sur individualiser son enseignement donc mes connaissances personnelles m'ont permis de commenter davantage le diaporama qui était proposé dans la ressource » $(\mathrm{F} 2)$.

Ici, le formateur met en valeur ce que lui apportent ses connaissances professionnelles dans la mise en œuvre de la formation. Il fait ainsi appel à des stratégies (Pastré, 2005) qui lui permettent de coordonner ses actions. L'importance de l'intégration de schèmes préétablis (Rabardel, 1995) s'affirme également dans une volonté de réaction aux propositions des stagiaires: "J'étais active surtout au niveau du contenu pédagogique parce que les stagiaires proposaient des scénarios et j'étais plus attentive au niveau de la construction des scénarios donc mon apport était surtout un apport théorique au niveau des scénarios et des questions pédagogiques quel est le rôle de l'enseignant par rapport à la balado-diffusion donc c'était surtout un apport pédagogique et donc d'observation sur la formation Pairform@nce»(F3). Enfin, dans son utilisation, le quatrième formateur (F4) dont l'expertise est moindre a ajouté quelques exemples personnels de l'utilisation du parcours, mais rien n'a été changé au parcours lui-même. Il a, par ailleurs, un avis peu favorable par rapport à la démarche Pairform@nce qui implique beaucoup de contraintes pour le concepteur et le formateur qui doivent décrire en détail le parcours et fractionner le contenu. $\mathrm{Du}$ point de vue d'un formateur également concepteur, les modifications peuvent être appréhendées dès le processus de conception (Folcher, 2003) :

«Donc, il peut y avoir des ajouts théoriques suivant le niveau du public. Il peut y avoir des ajouts d'activités parce qu'on ouvre le logiciel à l'utilisation individuelle et collective alors pour le prof de maths qui utilise le parcours, il peut y avoir des ajouts de séquences, séances » $(\mathrm{F} 1)$.

Il nous semble alors que, d'une part, les contraintes d'ordre organisationnel et de gestion humaine modifient l'activité d'appropriation du parcours de formation et participent à son instrumentation et que, d'autre part, les modifications liées à une évaluation personnelle du parcours modifient le parcours, notamment par l'ajout de séquences pédagogiques ou de nouvelles ressources.

Ces éléments nous semblent intégrer les dimensions de l'instrumentation et de l'instrumentalisation (Rabardel, 1995). En effet, l'appropriation passe par :

- l'instrumentation des ressources que le formateur aura adaptées à ses besoins et au contexte d'utilisation de l'académie et de la formation; ce qui impliquera une modification du scénario prescrit et une utilisation différente des assistants proposés avec le parcours (calendrier, étapes). Cette instrumentation des ressources est associée à une prise en compte des facteurs externes décrits ci-dessus ;

- mais également par l'instrumentalisation du parcours par l'ajout, la modification et la suppression de ressources.

De manière spécifique, l'analyse de ces entretiens permet de mettre en évidence des indicateurs d'instrumentation, qui ne sont pas sans faire appel aux quatre facteurs externes que nous avons décrits ci-dessus 5 .

— L'adaptation aux potentialités et contraintes de l'artefact (Trouche, 2007) ;

— La temporalité d'appropriation ;

— L'adaptation au contexte d'appropriation ;

— L'expertise du formateur.

Pour les déterminer, on se demandera si les prescriptions temporelles, humaines pour

5 Nous différencions ainsi les facteurs externes en tant que tel et les modifications qu'elles impliquent dans et par l'activité du formateur. 
l'organisation et les prescriptions techniques du concepteur ont été rigoureusement suivies, et si elles l'ont été, ce que cela a impliqué pour l'activité du formateur et si elles ne l'ont pas été, pour quelles raisons et quelle activité a alors été menée.

Les indicateurs d'instrumentalisation sont attachés à une évaluation personnelle du parcours de formation, ils sont construits en termes d'acceptabilité personnelle et sociale, laquelle est prise en compte par l'utilisateur. Ils s'observent notamment en matière de transformation des ressources et peuvent dépendre des contraintes d'instrumentation. Les médiations instrumentales proposées par Rabardel (1995) apparaissent alors comme un indicateur du produit de l'appropriation :

- La médiation épistémique (l'instrument est un moyen qui permet la connaissance de l'objet) de l'activité met en valeur le lien entre le formateur et le parcours de formation. Il précise la capabilité (aptitude) soit l'appel à des schèmes pré-requis du formateur face à ce parcours et la formation qu'il doit mettre en œuvre avant l'activité en fonction de schèmes d'utilisation précédemment utilisés. On se demandera alors quelles sont les compétences techniques prescrites par le concepteur du parcours, quelles sont les compétences techniques réelles du formateur et si ce dernier a modifié ou restreint l'utilisation prescrites des ressources compte tenu de ses compétences déjà acquises ou à acquérir?

— La médiation pragmatique (l'instrument est moyen d'une action transformatrice dirigée vers l'objet) de l'activité appréciera des modifications du parcours dues à l'instrumentation du parcours, quand celle-ci ne suffit plus. On recherchera alors quelles modifications ont été apportées au parcours dans l'utilisation des ressources compte tenu de ce nouveau contexte?

- La médiation heuristique (l'instrument est moyen d'une action transformatrice dirigée vers le sujet-même) implique une certaine critique des ressources proposées compte tenu d'un habitus du même type de parcours ou ressources. On demandera alors pour quelles raisons ces modifications ont été apportées et si elles font référence à une autre expérience du même type précédemment effectuée ?

- La médiation collaborative (l'instrument est moyen d'une action transformatrice dirigée vers l'action de l'autre sujet) implique l'évaluation du parcours mis en œuvre de manière collaborative, c'est-à-dire en fonction des autres sujets. On se demandera si les ressources ont été modifiées compte tenu des autres sujets intégrés de manière directe ou indirecte dans l'activité ${ }^{6}$.

Ce cadre théorique nous permet ainsi de mettre en valeur des indicateurs d'appropriation.

\section{6.- Analyse principale : Journaux de Bord des Formateurs}

\section{1.- Méthode spécifique à cette analyse}

Notre objectif est maintenant de vérifier que les indicateurs d'appropriation proposés apparaissent bien dans l'appropriation d'un parcours de formation toujours dans le cadre du dispositif Pairform@nce. Pour ce faire, nous avons analysé deux journaux de bord papier rempli par deux autres formateurs que ceux interviewés dans la première phase de cette étude. Ces formateurs ont une connaissance experte du dispositif Pairform@nce, car ils sont concepteurs d'un autre parcours.

Un journal de bord est constitué d'un carnet de bord, rempli de manière chronologique, mais également d'une annexe qui recense l'utilisation et la modification des ressources proposées

6 En ce qui concerne Pairform @ nce, il s'agira surtout des stagiaires à qui est destinée la formation et également des autres formateurs, avec qui l'appropriation d'un parcours peut être partagée. 
par le parcours. L'intérêt de tels documents, c'est qu'ils permettent d'obtenir des traces d'une appropriation en « train de se faire ».

À la fin de chacun des journaux, trois questions sont posées aux formateurs : (1) quelles ont été les contraintes liées à la gestion et à l'organisation de la mise en œuvre du parcours dans un cadre institutionnel précis, celui de l'organisation des stages par les académies; (2) quelles ont été les contraintes techniques et (3) quelles évaluations ont été faites du parcours par les formateurs pour cette mise en œuvre?

Pour cette étude, nous avons choisi de centrer l'analyse sur l'utilisation des ressources proposées pour enrichir le parcours de formation par le concepteur en analysant les discours écrits par les formateurs (Tableau 1). Les modifications, ajouts et suppressions exprimées par les formateurs ont été classifiés selon les indicateurs définis dans la première partie de l'étude.

\begin{tabular}{|c|c|c|c|c|}
\hline INSTRUMENTATION & \multicolumn{4}{|c|}{$\begin{array}{l}\text { INTRUMENTALISATION } \\
\text { Par évaluation de la médiation }\end{array}$} \\
\hline $\begin{array}{l}\text { Contraintes } \\
\text { organisationnelles } \\
\text { Et/ou } \\
\text { Contraintes } \\
\text { techniques, juridiques }\end{array}$ & $\begin{array}{l}\text { épistémique : } \\
\text { (connaissance de } \\
\text { l'objet) } \\
\text { Evaluation et } \\
\text { modifications liées } \\
\text { aux compétences et } \\
\text { à la capabilité }\end{array}$ & $\begin{array}{l}\text { pragmatique } \\
\text { (transformation } \\
\text { de l'objet): } \\
\text { Evaluation et } \\
\text { modifications } \\
\text { issues des } \\
\text { contraintes } \\
\text { organisationnell } \\
\text { es et/ou } \\
\text { techniques }\end{array}$ & $\begin{array}{l}\text { heuristique } \\
\text { (transformation du } \\
\text { sujet): } \\
\text { Evaluation et } \\
\text { modifications issues } \\
\text { d'un " habitus » } \\
\text { d'utilisation du } \\
\text { parcours }\end{array}$ & $\begin{array}{l}\text { collaborative } \\
\text { (action } \\
\text { transformatrice } \\
\text { vers l'action de } \\
\text { l'autre sujet) : } \\
\text { Evaluation et } \\
\text { modifications liées } \\
\text { aux autres sujets } \\
\text { impliqués dans } \\
\text { l'utilisation du } \\
\text { parcours. }\end{array}$ \\
\hline $\begin{array}{l}3 \text { jours prévus dans le } \\
\text { parcours, } 2 \text { jours } \\
\text { accordés puis un } 3^{\text {ème }} \\
\text { jour ajouté }\end{array}$ & $\begin{array}{l}\text { Exemples et TP } \\
\text { adaptés } \\
\text { Ressources autres } \\
\text { que Géogebra et } \\
\text { TEP écartées } \\
\text { Restriction de } \\
\text { l'usage des } \\
\text { assistants de } \\
\text { formation. }\end{array}$ & $\begin{array}{l}\text { Calendrier } \\
\text { proposé non } \\
\text { utilisé car le } \\
\text { stage prévu avec } \\
3 \text { présentiels n'a } \\
\text { obtenu que } 2 \\
\text { présentiels }\end{array}$ & $\begin{array}{l}\text { Les ressources } \\
\text { proposées ne } \\
\text { pointent pas } \\
\text { suffisamment sur } \\
\text { l'aspect } \\
\text { individualisation } \\
\text { Utilisation des } \\
\text { ressources élaborées } \\
\text { par l'équipe Nancy- } \\
\text { Metz } \\
\text { Utilisation de } \\
\text { ressources perso } \\
\text { Utilisation de mode } \\
\text { d'emplois issus } \\
\text { d'une autre } \\
\text { formation } \\
\text { Utilisation du } \\
\text { diaporama C2m@tic } \\
\text { individualisation } \\
\text { Exemples de TP et } \\
\text { séances par les } \\
\text { formateurs ajoutés }\end{array}$ & $\begin{array}{l}\text { Références } \\
\text { bibliographiques } \\
\text { sur l'emploi de } \\
\text { BEL non } \\
\text { proposées aux } \\
\text { stagiaires } \\
\text { Assistants de } \\
\text { formation non } \\
\text { proposés aux } \\
\text { stagiaires. } \\
\text { Diaporama } \\
\text { d'introduction non } \\
\text { utilisé car } \\
\text { correspond à une } \\
\text { mise en œuvre } \\
\text { Diaporama } \\
\text { «individualisation } \\
\text {-différenciation » } \\
\text { non utilisé }\end{array}$ \\
\hline
\end{tabular}

Tableau 1 : Exemples d'indicateurs d'appropriation des ressources observés dans les journaux de bord des formateurs pour la mise en œuvre de parcours dans Pairform@nce

Table 1: Examples of resource appropriation indicators based on the analysis of logbooks completed by teachers using scenarios in Pairform@nce 


\section{2.- Résultats}

Les indicateurs d'appropriation que nous avons déterminés par l'analyse des entretiens peuvent être validés par cette analyse. En effet, de manière globale, l'appropriation se construit en fonction de facteurs externes d'où l'instrumentation des ressources d'une part, et d'autre part, elle se construit en fonction de la médiation qui opère entre le sujet et ces facteurs externes d'où l'instrumentalisation des parcours de formation.

\subsection{1.- Indicateurs de l'instrumentalisation des parcours}

Les formateurs ont instrumentalisé le parcours et ont modifié l'utilisation des ressources prescrites : ils se le sont approprié et l'ont mis en œuvre dans leurs stages. Nous avons pu vérifier avec cette analyse que ces indicateurs d'appropriation et d'instrumentalisation sont bien attachés à une évaluation personnelle du parcours. Nous avons ainsi retrouvé des médiations épistémiques, pragmatiques, heuristiques et collaboratives dans chacun des parcours appropriés. L'évaluation de la médiation épistémique passe par des modifications de ressources afin d'alléger le parcours; certaines ont été adaptées aux compétences du formateur, d'autres ont été écartées, car le formateur s'est focalisé sur un aspect du parcours. L'évaluation de la médiation pragmatique a consisté en une réorganisation des stages compte tenu des obstacles et difficultés organisationnelles et donc à la non-utilisation du calendrier proposé. L'évaluation de la médiation heuristique a impliqué l'utilisation de ressources extérieures au parcours comme par exemple des ressources utilisées lors d'une autre formation, voire même d'un transfert du parcours sur une autre plateforme mieux connue des formateurs et correspondant, selon eux, en tout point à l'esprit du parcours proposé. Enfin, l'évaluation de la médiation collaborative a impliqué un choix précis de ressources proposées aux stagiaires.

Notons que ces différentes évaluations fonctionnent ensemble et semblent être partie prenante de la genèse instrumentale. Les évaluations épistémiques et pragmatiques de l'utilisation de ressources associées à un parcours de formation sont directement en lien avec l'évaluation collaborative des ressources. La médiation épistémique a impliqué l'utilisation des assistants de formation pour les formateurs et non pour les stagiaires, car jugés trop complexes et inutiles par les formateurs. L'évaluation de la médiation épistémique du parcours semble donc être liée à l'évaluation collaborative de celui-ci.

Qui plus est, l'évaluation de la médiation heuristique du parcours a comme conséquence une réévaluation de la médiation collaborative. Ainsi, l'utilisation d'un logiciel de mathématiques dans un cadre personnel, c'est-à-dire sans lien avec le parcours et la pratique de formation au sein de Pairform@nce, implique une évaluation de la médiation heuristique qui sera rapidement traduite au niveau collaboratif, c'est-à-dire que le formateur propose à ses stagiaires d'insister sur l'aspect didactique du logiciel quand ils l'utiliseront avec leurs élèves.

Par ailleurs, le formateur semble s'approprier les ressources proposées dans le parcours de formation par deux types d'activités, généralement non dissociables dans le temps :

— Des activités qui impliquent davantage l'évaluation des médiations épistémiques (pour lui-même) et heuristiques dans un contexte élargi, celui qui l'entoure. Ce qui peut correspondre à ce que nous proposions en deuxième étape : c'est-à-dire l'appropriation du parcours par la maitrise des dimensions techniques du parcours, l'évaluation de l'importance cognitive, psychologique et langagière de l'intégration de la technologie dans ce parcours proposé et enfin par l'intégration du parcours dans ses pratiques quotidiennes. Pour ce faire, il se rattache à ses «habitus», ses habitudes de travail dans d'autres contextes et fait appel à des schèmes d'action qu'il peut intégrer à cette activité. Ces activités se rattachent plus particulièrement à une mise en œuvre individuelle du parcours.

— Des activités qui impliquent davantage l'évaluation des médiations épistémiques (pour 
les stagiaires), pragmatiques dans un contexte donné, celui de la formation à mener et donc collaboratives. Le formateur s'approprie le parcours en évaluant le contexte organisationnel de la formation et utilise la technologie comme modèle pour ses stagiaires afin de lui donner une signification sociale pour justifier sa formation. Ces activités se rattachent davantage à une mise en œuvre sociale du parcours.

\subsection{2.- Indicateurs de l'instrumentation des parcours}

- Contexte (adaptation compte tenu $d u$...)

La lecture des journaux de bord montre que l'activité des formateurs a été modifiée et a dû être adaptée en fonction de facteurs externes; souvent des contraintes organisationnelles et techniques dans les deux parcours. Il y a ainsi eu instrumentation. Dans ce cas, les indicateurs de modifications issus de contraintes organisationnelles sont liés à un fossé entre les prescriptions du concepteur et les décisions organisationnelles du Plan Académique de Formation, c'est-à-dire le contexte. Ainsi, on observe que l'organisation des temps en présence a impliqué des difficultés pour les groupes de formateurs, qu'elles soient techniques ou organisationnelles.

- Expertise

Il semble intéressant de noter que chacun des deux parcours a été découvert et utilisé par des formateurs qui sont également concepteurs d'un autre parcours. De fait, la démarche technique de Pairform@nce était parfaitement maitrisée par ces deux formateurs et les deux groupes avaient un certain nombre de connaissances techniques propres à l'intégration des TICE dans l'activité d'enseignement. Cela explique probablement pourquoi les contraintes techniques que nous supposions dans le cadre de la préparation de la mise en œuvre n'ont pas été un obstacle majeur à l'appropriation du parcours. En revanche, cette remarque est l'occasion de confirmer un indicateur d'instrumentation que nous avions déterminé en première partie de cette étude, l'expertise des formateurs, comme expérience du métier de formateur et dans ce cas précis, expérience du métier de formateur intégrant des TICE, expérience qui s'est construite sur une expérience de concepteur.

- Temporalité

L'étude des journaux de bord papier nous permet de mieux évaluer le facteur externe de l'appropriation qu'est la temporalité d'usage. Rappelons que le temps est un vecteur d'innovation (Paquelin, 2009) et que le modèle de Depover et Strebelle (1997) appelé Modèle systémique de l'innovation présente trois niveaux d'intégration d'une innovation, largement associé au facteur temporel :

— L'adoption renvoie à un changement dans sa pratique soit par conviction personnelle, soit sous une pression externe.

— L'implantation rend compte du changement de pratique opéré durant la phase d'adoption et qui se caractérise par des traces visibles d'activités pédagogiques innovantes ayant un impact sur l'environnement.

— La routinisation fait référence à une utilisation régulière des nouvelles pratiques sans support externe.

On observe, ici, la temporalité d'usage dans le cadre de l'appropriation d'un seul parcours de formation, c'est-à-dire dans le cadre du journal de bord papier rempli à cette occasion. Par définition, le journal de bord est rempli de manière chronologique et il est présenté comme tel. Le premier journal de bord a été rempli sur une période s'étalant entre le 30 septembre 2008 et le 16 mars 2009. Le deuxième journal de bord analysé a été rempli entre le 18 décembre 2008 et le 15 mai 2009.

Afin de mieux observer le lien entre la temporalité et l'appropriation, nous avons établi des niveaux de modifications qui ont été ensuite appliqués au journal de bord chronologique (Tableau 2) : 


\begin{tabular}{|c|c|c|c|c|}
\hline \multicolumn{2}{|c|}{ Journal de bord $n^{\circ} 1$} & \multicolumn{2}{|c|}{ Journal de bord $n^{\circ} 2$} & \multirow{2}{*}{$\begin{array}{l}\text { Analyse : } \\
\text { modifications }\end{array}$} \\
\hline Mois & Activités & Mois & Activités & \\
\hline Septembre 2008 & $\begin{array}{l}\text { Echanges de mails } \\
\text { Réunions (visio- } \\
\text { conférence) }\end{array}$ & Décembre 2008 & $\begin{array}{l}\text { Echanges de mails } \\
\text { Consultation du } \\
\text { parcours } \\
\text { Parcours intéressant } \\
\text { mais trop dense } \\
\text { Lecture et évaluation } \\
\text { des ressources } \\
\text { proposées }\end{array}$ & $\begin{array}{l}\text { Découverte du } \\
\text { parcours. Pas ou } \\
\text { peu de } \\
\text { modification } \\
\text { directe. } \\
\text { Modifications de } \\
\text { niveau } 1\end{array}$ \\
\hline Octobre 2008 & $\begin{array}{l}\text { Echange de mail } \\
\text { Réunions } \\
\text { Adaptation de l'ordre du } \\
\text { jour + calendrier } \\
\text { Adaptation du diaporama }\end{array}$ & Janvier 2009 & $\begin{array}{l}\text { Lecture des } \\
\text { documents } \\
\text { Prise en main du } \\
\text { logiciel (lecture doc } \\
\text { prise en main) } \\
\text { Retour sur les étapes } \\
2 \text { et } 3\end{array}$ & $\begin{array}{l}\text { Plusieurs } \\
\text { adaptations dans } \\
\text { la préparation } \\
\text { des ressources } \\
\text { (diaporama et } \\
\text { calendrier) } \\
\text { Modifications de } \\
\text { niveau } 3\end{array}$ \\
\hline Novembre 2008 & $\begin{array}{l}\text { Présentiel } 1 \\
\text { Rédaction du compte } \\
\text { rendu } \\
\text { Relance par mail des } \\
\text { stagiaires } \\
\text { Mise en place du travail } \\
\text { collaboratif }\end{array}$ & Février 2009 & $\begin{array}{l}\text { Premier présentiel } \\
\text { Essais des stagiaires } \\
\text { Lecture et révision } \\
\text { du diaporama } \\
\text { Demande d'une aide } \\
\text { technique }\end{array}$ & $\begin{array}{l}\text { Plusieurs } \\
\text { adaptations dans } \\
\text { la préparation } \\
\text { des ressources } \\
\text { (diaporama ) } \\
\text { Adaptation } \\
\text { organisationnelle } \\
\text { Modifications de } \\
\text { niveau } 3\end{array}$ \\
\hline Décembre 2008 & $\begin{array}{l}\text { Préparation Présentiel } 2 \\
\text { Adaptation ordre du jour } \\
\text { Réunions visio- } \\
\text { conférence }\end{array}$ & Mars 2009 & $\begin{array}{l}\text { Téléchargement du } \\
\text { travail des stagiaires } \\
\text { Echanges de mails } \\
\text { avec les responsables } \\
\text { de la formation } \\
\text { Préparation } \\
\text { deuxième présentiel } \\
\text { Deuxième présentiel } \\
\text { Retard car problème } \\
\text { de gestion de salles }\end{array}$ & $\begin{array}{l}\text { Une ou plusieurs } \\
\text { adaptation } \\
\text { organisationnelle } \\
\text { (ordre du jour) } \\
\text { Modifications de } \\
\text { niveau } 2\end{array}$ \\
\hline Janvier 2009 & $\begin{array}{l}\text { Travail sur ordre du jour } \\
\text { Présentiel } 2 \\
\text { Analyse séances des } \\
\text { stagiaires }\end{array}$ & Avril 2009 & $\begin{array}{l}\text { Préparation } 3^{\text {ème }} \\
\text { journée de présentiel } \\
\text { Récupération des } \\
\text { fichiers des } \\
\text { stagiaires }\end{array}$ & $\begin{array}{l}\text { Modifications de } \\
\text { niveau } 5 \\
\text { Adaptation des } \\
\text { ressources }\end{array}$ \\
\hline
\end{tabular}

Tableau 2 : Synthèse des activités des formateurs répertoriées dans les journaux de bord papier

Table 2: Summary of the teachers' work as recorded in paper logbooks

La première partie du Tableau 2 met en évidence l'aspect temporel du processus d'appropriation qui modifie les activités d'appropriation. Dans un premier temps, les activités des formateurs se consacrent en particulier à la lecture, à la découverte des parcours et à l'évaluation des ressources. La prise de contact par l'échange de mails est également un aspect évoqué de manière plus prononcée au début des journaux de bord. Dans un second temps, les parcours sont modifiés dans l'objectif de l'organisation des séances en présence. Dans un troisième temps, les formateurs « reviennent » vers le parcours pour de nouveau lire les ressources et enfin de nouveau les modifier en fonction des contextes. Toutefois, les niveaux de modification des parcours ne s'amplifient pas en fonction de la temporalité 
(Figure 1):

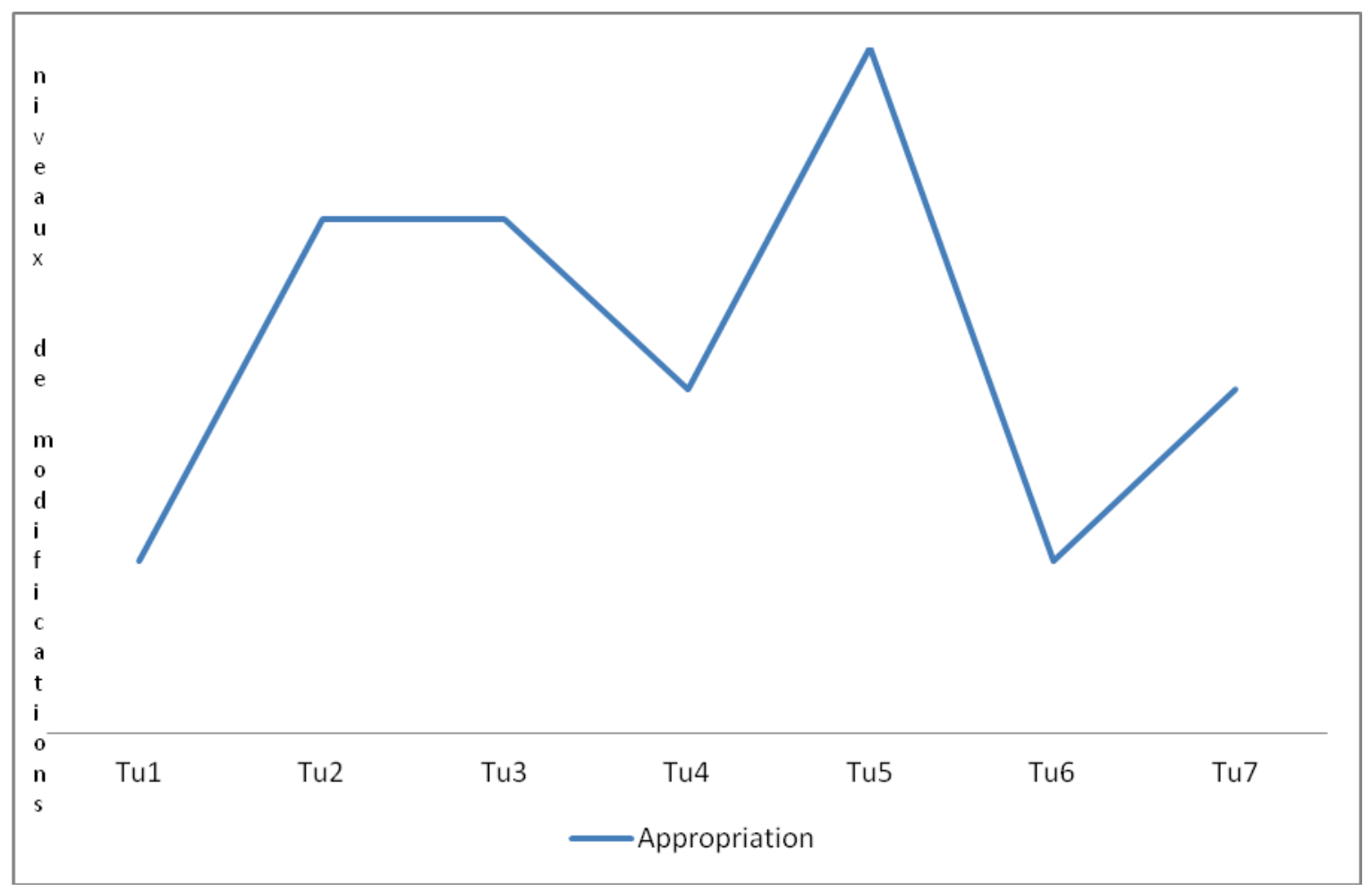

Figure 1 : Modifications et temporalité d'usage

Figure 1: Modifications and temporality of use

Si le processus d'appropriation d'un parcours de formation évolue en fonction d'une temporalité d'usage spécifique dans la mesure où les modifications interviendront plus dans un deuxième temps (après avoir pris connaissance du parcours), les résultats de cette analyse valident l'idée que l'appropriation dépend également de facteurs externes: ici, les formateurs ont particulièrement modifié les parcours au moment de la préparation des présentiels.

Cependant, si ces journaux de bord papier nous permettent d'observer des récurrences dans la temporalité d'appropriation de parcours de formation, ils n'ont été proposés qu'à des experts.

De manière générale, il nous semble que la temporalité d'usage, les facteurs externes et le niveau d'expertise ont un impact sur la genèse instrumentale de l'artefact et de manière plus générale sur le processus d'appropriation. Le processus d'appropriation, non seulement, intègre ces trois facteurs, mais aussi, en tant que genèse instrumentale «en cours d'accomplissement », se construit selon ces trois aspects.

\section{7.- Discussion et recommandation}

L'appropriation des ressources proposées dans le parcours de formation implique un décalage entre l'usage prescrit de ces ressources et l'usage effectué réellement par le formateur. Ce décalage peut être mis en évidence grâce à l'analyse des différentes évaluations mises en œuvre dans la genèse instrumentale et l'appropriation du parcours.

Cette hypothèse a pu être vérifiée par l'analyse des journaux de bords papier remplis par des équipes de formateurs intégrés au Programme Pairform@nce. Nous avons, à partir de cette analyse, remarqué que certaines médiations étaient impliquées ensemble dans l'activité. Nous avons donc déterminé deux groupes de médiations. Ainsi, ce regroupement des 
indicateurs selon deux types d'activités fonctionne, dans la mesure où nous avons choisi de différencier une appropriation dite individuelle du parcours de formation et une appropriation en contexte ou sociale du parcours proposé et que ces deux types d'activités, pouvant être menés conjointement, représentent une grande partie de l'activité du formateur telle que nous avons pu le vérifier dans les journaux de bord. Cette structuration par analyse de différentes médiations répond par ailleurs aux problématiques individuelles et collectives du processus d'appropriation.

Néanmoins, cette analyse, par la mise en relief des évaluations de médiations qui nous permet de déterminer des grandes familles d'indicateurs d'appropriation, ne peut pas être généralisable. L'interprétation et l'outil que nous avons proposés sont le fruit d'une analyse qualitative et s'appuient sur les remarques des formateurs à qui la question a été posée. Les données dont nous disposions pour mener à bien cette analyse sont précieuses, mais ne portaient que sur deux utilisations de deux parcours de formation.

Dans cette perspective, notre prochain objectif est de développer un outil «Journal de Bord en Ligne », un « objet intermédiaire » (Vinck, 1999) de l'appropriation, qui permettra aux formateurs de consigner leur activité de manière plus systématique et ainsi (1) de (in)valider de manière plus générale nos indicateurs et (2) d'approfondir l'analyse de l'activité d'appropriation en tentant de mettre à jour des patterns d'action liés à chaque type de médiation.

Le Journal de Bord en ligne est ainsi un outil destiné à trois types d'utilisateurs: le formateur, le concepteur et le chercheur. Il est accessible via un compte sur une plateforme collaborative. S'il est bien vrai que la collaboration prescrite n'est pas directe, car elle n'implique pas de communication entre les différents utilisateurs, elle propose néanmoins à chacun des utilisateurs d'être informé des actions des autres. La collaboration indirecte (Katz, 1999) s'impose ici puisque les différents utilisateurs effectuent des tâches tout à fait différentes bien que leurs activités soient facilement comparées. Cette dernière permet également de faire du Journal de Bord, une proposition pour «un monde commun» plus qu'une « autorité » (Béguin, $2005 ; 2007$ )

Par ailleurs, une collaboration indirecte, au même titre qu'une collaboration directe est un support à la réflexivité si elle est mobilisatrice de traces (Ollagnier-Beldame, \& Mille, 2009) d'où la proposition d'un outil commun à ces trois acteurs de la formation. Il permettrait d'évaluer le parcours prescrit non seulement en termes d'utilité (par l'évaluation du formateur qui se l'est approprié), mais également en termes d'utilisabilité.

Enfin, il s'agit également de créer une communauté de pratique (Wenger, 1998) au sein du programme de formation Pairform@nce afin de mutualiser l'usage des parcours de formation et favoriser un processus d'apprentissage collaboratif et de conception dans l'usage.

Nous proposons donc ce Journal de Bord pour accompagner l'appropriation du parcours de formation sur la plateforme et permettre un retour à propos de l'adaptabilité du parcours :

- en fonction de différents facteurs externes: contextes, expertise d'appropriation, temporalité d'appropriation ;

— en fonction des médiations entretenues par les formateurs vis-à-vis de l'objet de l'activité via l'artefact qui est devenu un instrument.

Le Journal de bord en ligne serait destiné à aider et favoriser l'appropriation dans la mesure où celle-ci pourrait laisser libre cours à une adaptation «individuante » du parcours sans pour autant dénaturer l'artefact prescrit.

Par ailleurs, le Journal de Bord en ligne pourrait également favoriser pour le formateur, une approche réflexive (Schön, 1987) de sa propre activité afin d'évaluer sa propre démarche face à un usage prescrit d'une ressource et améliorer son appropriation des parcours. En 
effet, la réflexivité participe à l'apprentissage dans un cadre professionnel et se construit dans le temps de l'action et de la pratique du professionnel. La pratique réflexive propose ainsi une activité métacognitive. Il s'agit d'une activité dont l'objectif est l'activité ellemême suscitant, de fait, une prise de conscience réfléchie du formateur tournée vers luimême. La mise en valeur de cette méta-activité passe ainsi par l'utilisation des traces d'activité, dans un format «qui doit faire sens pour les humains (utilisateurs et/ou analyste) »(Ollagnier-Beldame, \& Mille, 2007 p. 2). Ces méthodes qui visent à l'analyse de l'activité d'appropriation du parcours seront préconisées par le Journal de bord que nous proposons, ainsi, composé de trois modules :

- Un module de saisie : destiné au formateur, celui-ci consigne son activité au fur et à mesure de la formation. Ce module se présente sous la forme d'un questionnaire et peut être également rempli par le concepteur qui peut ainsi proposer de façon précise les activités prescrites. On peut imaginer que le formateur ait accès au Journal de Bord du concepteur : ce qui participe à notre proposition de collaboration indirecte.

— Un module de visualisation : destiné au formateur pour la réflexivité, au concepteur et au chercheur pour l'amélioration des prescriptions. Il représente graphiquement, de façon paramétrée, les activités du formateur.

- Un module d'analyse : destiné au chercheur, car l'activité est décrite selon des catégories afin de mettre en valeur les moyens mis en œuvres pour adapter la formation à son contexte.

Ce Journal de Bord devrait également permettre d'avancer dans nos recherches, notamment de tracer plus de données, ce qui apportera des éclairages quant à son utilité et son utilisabilité. En effet, la saisie de données sur sa pratique et de manière régulière n'est pas aisée et il est nécessaire de réfléchir aux fonctionnalités alliant rapidité d'usage et complétude des informations pour le formateur et le concepteur dans le cadre d'un processus dialogique d'apprentissage mutuel (Béguin, 2004).

\section{8.- Conclusion}

Nous avons montré que la théorie de la genèse instrumentale (Rabardel, 1995) pouvait éclairer non seulement la définition du processus d'appropriation, mais aider à définir des indicateurs qui permettent de l'observer plus finement.

Quand un formateur s'approprie un scénario pédagogique ou parcours de formation dont il n'est pas le concepteur, il opère donc une genèse instrumentale :

— Individuante, cette genèse instrumentale est «liée » à un certain nombre de facteurs que nous avons mis en valeur.

- Au sein de cette genèse instrumentale, le formateur opère un certain nombre de médiations qui sont «actives» dans le processus d'appropriation (épistémique, pragmatique, heuristique, collaborative).

- La genèse instrumentale pourrait être prise en compte dans la conception des scénarios ou parcours. L'outil Journal de Bord en Ligne participerait alors à une genèse professionnelle (Béguin, 2005) du travail, proposée au sein d'un dispositif : «Les genèses professionnelles concernent le développement, par le sujet, des ressources de son action: la constitution des instruments (comme durant les genèses instrumentales), mais aussi de beaucoup d'autres aspects, tels que les développements des conceptualisations et compétences (Pastré, 1999), ainsi que des formes organisées de l'action au sein des collectifs » (Béguin, 2007, p. 5) voire même à une Communauté de Pratique (Wenger, 1998)

Si le contexte de formation continue des enseignants est moins « contraignant » que d'autres corps de métier étudiés dans la littérature scientifique en ergonomie (aviation, etc.), il n'en 
reste pas moins qu'un seuil d'appropriation reste nécessaire afin que :

- la mutualisation des scénarios pédagogiques (ou parcours de formation) garde tout son sens ;

— cette mutualisation puisse favoriser la créativité des formateurs.

Ce seuil d'appropriation pourrait ainsi se définir selon des «genèses professionnelles ». Le Journal de Bord en ligne que nous préconisons serait un outil d'aide à ces dernières.

\section{BIBLIOGRAPHIE}

Béguin, P. (2004). L'ergonome acteur de la conception. In P. Falzon (Ed.), Ergonomie (pp. 375-390). Paris: PUF

Béguin, P. (2005). Concevoir pour les genèses professionnelles. In P. Rabardel, \& P. Pastré (Eds.), Modèles du sujet pour la conception (pp. 31-52). Toulouse: Octarès Edition

Béguin, P. (2007). Innovation et cadre sociocognitif des interactions concepteurs-opérateurs : une approche développementale. Le travail humain, 70(4), 369-390. DOI : 10.3917/th.704.0369.

Brassard, C., \& Daele, A. (2003). Un outil réflexif pour concevoir un scénario pédagogique intégrant les TIC. In C. Desmoulins, P. Marquet, \& D. Bouhineau (Eds.), EIAH 2003, Strasbourg, INRP.

Charlier, B., Deschryver, N., \& Peraya, D. (2006). Apprendre en présence et à distance. Une définition des dispositifs hybrides. Distances et savoirs, 4, 469-496.

Cotreaux, S., \& Jacques, E. (2004). L'usage dans la conception des dispositifs technologiques d'apprentissage. In ISDM (Ed.), Colloque TICE Méditerranée (p. 11). Nice. http://isdm.univtln.fr/PDF/isdm18/37-jacques-cotreaux.pdf

Cuvelier, L., \& Caroly, S. (2009). Appropriation d'une stratégie opératoire : un enjeu du collectif de travail. Activités, 6(2), 57-74. http://www.activites.org/v6n2/v6n2.pdf

Depover, C., \& Strebelle, A. (1997). Un modèle et une stratégie d'intervention en matière d'intégration des TIC dans le processus éducatif. In L.-O. Pochon, \& A. Blanchet (Eds.), L'ordinateur à l'école : de l'introduction à l'intégration (pp. 73-98). Neuchâtel \& Lausanne: IRDP \& LEP.

Folcher, V. (2003). Appropriating artifacts as instruments: when design-for-use meets design-in-use. Interacting with Computers, 15(5), 647-663.

Haradji, Y., \& Faveaux, L. (2006). Évolution de notre pratique de conception (1985-2005) : modéliser pour mieux coopérer à partir des critères d'utilité, d'utilisabilité. @ ctivités, 3(1), 67-98. http://www.activites.org/v3n1/haradji.pdf

Katz, L. (1999). The Potential of Modern Telelearning Tools for Collaborative Learning. Canadian Journal of Communication, 24 (3). http://www.cjconline.ca/index.php/journal/article/view/1115/1021

Mailles-Viard Metz, S. (2009). Les situations d'apprentissage collectives instrumentées dans le supérieur : identification et exploration, évidences et tendances. Les enjeux de l'information et de la communication. [En ligne] http://w3.u-grenoble3.fr/les_enjeux/2008supplement/Metz/index.php

Michel, C., Garrot, E., \& George, S. (2007). Situations d'apprentissage collectives instrumentées : Étude de pratiques dans l'enseignement supérieur. Distances et savoirs, 5(4), 527-546.

Ollagnier-Beldame, M., \& Mille, A. (2007). Faciliter l'appropriation des EIAH par les apprenants via les traces informatiques d'interactions. Rapport de recherche RR-LIRIS-2007-023.

Ollagnier-Beldame, M., \& Mille, A. (2009). E-learning : Tracer l'activité des apprenants pour favoriser leur appropriation des systèmes. Revue du Management Technologique, 15(3).

Paquelin, D. (2009). L'appropriation des dispositifs numériques de formation : du prescrit aux usages. Paris: L'Harmattan.

Pastré, P. (1999). Apprendre en situation. Éducation permanente, 139, 13-36.

Pastré, P. (2005). La conception de situations didactiques à la lumière de la théorie de la conceptualisation dans l'action. In P. Rabardel, \& P. Pastré (Eds.), Modèles du sujet pour la conception : dialectiques activités développement (pp.73- 108). Toulouse: Octarès Editions.

Proulx, S. (2001). Les formes d'appropriation d'une culture numérique comme enjeu d'une société du 
savoir. Montréal.

http://www.acgrenoble.fr/ien.bourgoinashnord/IMG/pdf_es_TUIC_Enjeux_et_modalites_de_mise _en_oeuvre.pdf

Rabardel, P. (1995). Les hommes et les technologies : approche cognitive des instruments contemporains. Paris: Armand Colin, Paris.

Rabardel, P. (1999). Le langage comme instrument ? Éléments pour une théorie instrumentale étendue. In Y. Clot (Ed.), Avec Vygotski (pp. 241-265). Paris: La Dispute. Paris.

Rabardel, P. (2005). Instrument, activité et développement du pouvoir d'agir. In Ph. Lorino, \& R. Teulier (Eds.), Entre connaissance et organisation : l'activité collective (pp. 251-265). Paris: Edition La Découverte.

Samurçay, R. (2005). Concevoir des situations simulées pour la formation professionnelle : une approche didactique. In P. Rabardel, \& P. Pastré (Eds.), Modèles du sujet pour la conception : dialectiques activités développement (pp.53-72). Toulouse: Octarès.

Schön, D. (1987). Educating the reflective practitioner. San Francisco, CA: Jossey-Bass.

Soury-Lavergne, S., \& Sanchez, E. (2009). Pairform@nce, conception de parcours de formation continue pour les enseignants. EPAL, Grenoble. [En ligne] http://w3.ugrenoble3.fr/epal/dossier/06_act/pdf/epal2009-soury-lavergne-sanchez.pdf

Theureau J. (2011). Appropriation 1, 2, 3 ou Appropriation, Incorporation \& 'Inculturation'. Conférence journée Ergo-Idf Appropriation \& Ergonomie, 16/06/11, Paris. [En ligne] http://www.coursdaction.fr/02-Communications/2011-JT-C136.pdf

Tricot, A., \& Nanard, J. (1998). Un point sur la modélisation des tâches de recherche d'informations dans le domaine des hypermédias. In A. Tricot, \& J.-F. Rouet, (Eds.), Les hypermédias, approches cognitives et ergonomiques (pp. 35-56). Paris: Hermès.

Trouche, L. (2007). Des artefacts aux instruments, une approche pour guider et intégrer les usages des outils de calcul dans l'enseignement des mathématiques. Actes de l'Université d'été de SaintFlour, pp. 265-290.

Trouche, L. (2008). Le travail collaboratif : conception et usages de ressources pour et par les professeurs. Journée Associations d'enseignants et travail collaboratif : quel modèle ? [En ligne] http://www.cndp.fr/dossiersie/65/ptidos65.asp/

Vinck, D. (1999). Les objets intermédiaires dans les réseaux de coopération scientifique. Revue Française de Sociologie, 40, 385-414.

Wenger, E. (1998). Communities of Practice: Learning, Meaning, and Identity. Cambridge: Cambridge University Press.

\section{RESUME}

Cet article a pour objet l'analyse de l'appropriation de ressources en ligne et de scénarios de formation par un formateur qui n'en est pas le concepteur. À partir de réflexions théoriques autour de la genèse instrumentale et des notions d'appropriation, nous tentons de mettre en évidence des indicateurs d'instrumentation et d'instrumentalisation à propos des médiations épistémiques, pragmatiques, heuristiques et collaboratives décrites par Rabardel (1995) dans une situation de formation continue en ligne des enseignants du second degré : le dispositif Pairform@nce. Au sein de ce dispositif, des concepteurs de parcours mettent à disposition des formations pour des formateurs académiques. Ces indicateurs sont proposés à partir de l'analyse de quatre entretiens auprès de formateurs, puis vérifiés à la lecture de journaux de bord d'autres formateurs. Les résultats de l'analyse nous amènent à proposer un outil collaboratif asynchrone permettant d'améliorer l'appropriation d'un parcours de formation par un formateur: le Journal de Bord en ligne du formateur. 


\section{MOTS-CLES}

Appropriation, Formation continue à distance, Concepteur, Formateur, Collaboration

\section{RÉFÉRENCEMENT}

Duthoit, E., \& Mailles-Viard Metz, S. (2012). Analyse de l'appropriation d'un parcours pédagogique numérique par un formateur : le cas du dispositif Pairform@nce. Activités, 9(1), 106-126, http://www.activites.org/v9n1/v9n1.pdf

Article soumis le 27 juillet 2011, accepté pour publication le 12 janvier 2012 